\title{
Single-shot spatiotemporal measurements of ultrashort THz waveforms using temporal electric-field cross correlation
}

\author{
N. H. Matlis, ${ }^{1, *}$ G. R. Plateau, ${ }^{1,2}$ J. van Tilborg,${ }^{1}$ and W. P. Leemans ${ }^{1}$ \\ ${ }^{1}$ Lawrence Berkeley National Laboratory, Berkeley, California 94720, USA \\ ${ }^{2}$ École Polytechnique, Palaiseau, France \\ *Corresponding author: NHMatlis@lbl.gov
}

\section{DISCLAIMER}

This document was prepared as an account of work sponsored by the United States Government. While this document is believed to contain correct information, neither the United States Government nor any agency thereof, nor the Regents of the University of California, nor any of their employees, makes any warranty, express or implied, or assumes any legal responsibility for the accuracy, completeness, or usefulness of any information, apparatus, product, or process disclosed, or represents that its use would not infringe privately owned rights. Reference herein to any specific commercial product, process, or service by its trade name, trademark, manufacturer, or otherwise, does not necessarily constitute or imply its endorsement, recommendation, or favoring by the United States Government or any agency thereof, or the Regents of the University of California. The views and opinions of authors expressed herein do not necessarily state or reflect those of the United States Government or any agency thereof or the Regents of the University of California. 


\title{
Single-shot spatiotemporal measurements of ultrashort THz waveforms using temporal electric-field cross correlation
}

\author{
N. H. Matlis, ${ }^{1, *}$ G. R. Plateau, ${ }^{1,2}$ J. van Tilborg,${ }^{1}$ and W. P. Leemans ${ }^{1}$ \\ ${ }^{1}$ Lawrence Berkeley National Laboratory, Berkeley, California 94720, USA \\ ${ }^{2}$ École Polytechnique, Palaiseau, France \\ *Corresponding author: NHMatlis@lbl.gov
}

Received June 7, 2010; revised October 8, 2010; accepted October 20, 2010;

posted October 20, 2010 (Doc. ID 129643); published December 6, 2010

\begin{abstract}
A new single-shot technique based on linear spectral interferometry between a temporally short reader pulse and a temporally long probe pulse is demonstrated for measuring the spatiotemporal phase and amplitude of an optical probe for use as an ultrafast diagnostic. The probe spatiotemporal field information is recovered, with a resolution set by the duration of the reader pulse, by applying a single Fourier transform operation to the interferogram image, without need of any reference data. The technique was used in conjunction with electro-optic sampling to measure waveforms of coherent, ultrashort THz pulses emitted by electron bunches from a laser-plasma accelerator with sub- 50 fs resolution. The presence of strong spatiotemporal coupling in the $\mathrm{THz}$ waveforms and of complex temporal electron-bunch structure was determined. (c) 2010 Optical Society of America OCIS codes: $\quad 190.1900,300.6495,300.6500$.
\end{abstract}

\section{INTRODUCTION}

Optical probing has long been an important tool for the characterization of ultrafast phenomena because of its versatility, sensitivity, and ability to resolve both spatial and temporal features with high resolution. Because of its simplicity, the configuration most commonly used to probe temporal dynamics is a multishot one in which the delay of a probe pulse shorter than the features to be resolved is scanned. However, this approach is impractical for experiments done at low repetition rates or those in which the signal varies significantly from shot to shot, motivating the development of techniques capable of recording temporal variations in a single shot. Such is the case for the field of THz-time-domain spectroscopy (TDS), which relies on optical mapping of the electric-field temporal waveforms of pulses of $\mathrm{THz}$ radiation via a technique known as electro-optic sampling (EOS) [1-4]. In EOS, the THz pulse induces a transient birefringence in an electro-optically active crystal, which results in a polarization rotation of a copropagating optical probe pulse. The rotation is then converted to a temporal amplitude modulation by a polarizer.

THz-TDS has seen a dramatic surge in recent years because of the utility of ultrashort THz pulses as probes in an increasingly wide range of applications. For some applications, such as the characterization of current dynamics in semi- and superconductors (e.g., [5-7]), a scanning configuration can be implemented successfully because of the use of highrepetition-rate $(\mathrm{kHz})$ lasers and the stability of the material response function. For many other applications, such as the diagnosis of electron-bunch durations in laser-plasma accelerators (LPAs) [8], single-shot detection is critical due to the large shot-to-shot variability and the low repetition rates of high-intensity laser systems. As a result, a large body of work has been done to develop single-shot, EOS-based optical tech- niques that satisfy the needs of these applications [2-4,9-18]. Each, however, has had significant limitations.

Single-shot EOS was first achieved by a technique named "spectral encoding," in which a chirped pulse was used to provide a mapping between time and wavelength [10]. Spectral encoding, however, was plagued by a loss of temporal resolution due to the modification of the time-wavelength mapping caused by the encoding process, which produced distortions in the measurement $[12,13,19]$. This shortcoming was later addressed by combining the chirped probe with a temporally short pulse in a second-harmonic cross-correlation geometry $[14,15]$, providing high temporal resolution in the tens of femtoseconds. However, this technique has the disadvantage that it requires high probe intensities and, thus, expensive amplified lasers in order to get adequate signal. In addition, because the temporal signal is encoded spatially and the probe laser is focused at the interaction, spatial information about the $\mathrm{THz}$ pulses is lost. As the THz pulses in many of these applications are few to single cycle, implying relative bandwidths of order unity, the focused $\mathrm{THz}$ waveforms can exhibit strong spatiotemporal coupling due to a large variation of the Gaussian beam parameters over the spectrum [20]. Failure to resolve the spatial variations can, therefore, result in a loss of critical information.

In this article, a new technique named temporal electricfield cross correlation (TEX) is presented that overcomes the previous limitations, allowing measurement of $\mathrm{THz}$ waveforms with high temporal resolution and simultaneously providing one-dimensional spatial information. TEX is based on the measurement of the linear cross correlation of a chirped probe with a compressed reader pulse using spectral interferometry. The full electric-field information of the optical probe, convolved with that of the short reader, is retrieved, allowing signals to be encoded onto either the phase or the 
amplitude of the probe, or both. This dual capability is not present in previous EO methods and makes TEX applicable to the measurement of a wide range of phenomena beyond EOS. Because the detection is linear, TEX can be implemented with low-cost, unamplified laser systems, and because it does not require focusing of the optical probe, spatial information can be recorded and retrieved. The temporal detection window is easily tunable in the several picosecond range by adjusting the chirp of the probe pulse, and the temporal resolution of the phase and amplitude retrieval is set by the duration of the short reader pulse, which convolves the signal. Implementation of TEX to measure waveforms of intense THz pulses from an LPA is also demonstrated. Electrons experiencing a dielectric transition (such as a metallic foil or a plasmavacuum boundary [21,22]) emit coherent transition radiation (CTR) in the THz regime. The temporal and spectral properties of the $\mathrm{THz}$ radiation are strongly correlated to the electron-bunch temporal structure, making it an ideal diagnostic for ultrashort electron bunches.

It should be noted that the ability to retrieve the full electric field of an optical probe is not unique to TEX, but is shared by other techniques, such as frequency domain holography (FDH) and single-shot supercontinuum spectral interferometry (SSSI) [23-25]. The key advantage of TEX over FDH and SSSI is that recovery of the probe amplitude and phase does not require any reference data and is obtained from the TEX interferograms by a single Fourier transform (FT) operation, making it significantly easier to use than the others. By contrast, FDH and SSSI provide the pure electric-field retrieval of the probe without convolution.

\section{EXPERIMENTAL SETUP}

Figure 1 shows the setup for TEX implemented on an LPA-based $\mathrm{THz}$ source $[1,21,26]$. Using $\sim 400 \mathrm{~mJ}, 800 \mathrm{~nm}$, 45 fs pulses focused into a $2 \mathrm{~mm}$ gas jet of helium, electron bunches with large energy spreads and subpicosecond bunch durations were produced, resulting in the generation of CTR in the range of $0-4 \mathrm{THz}$. A portion of the radially polarized cone of THz emission was asymmetrically sampled by collection with an off-axis parabola (OAP). The resulting collimated

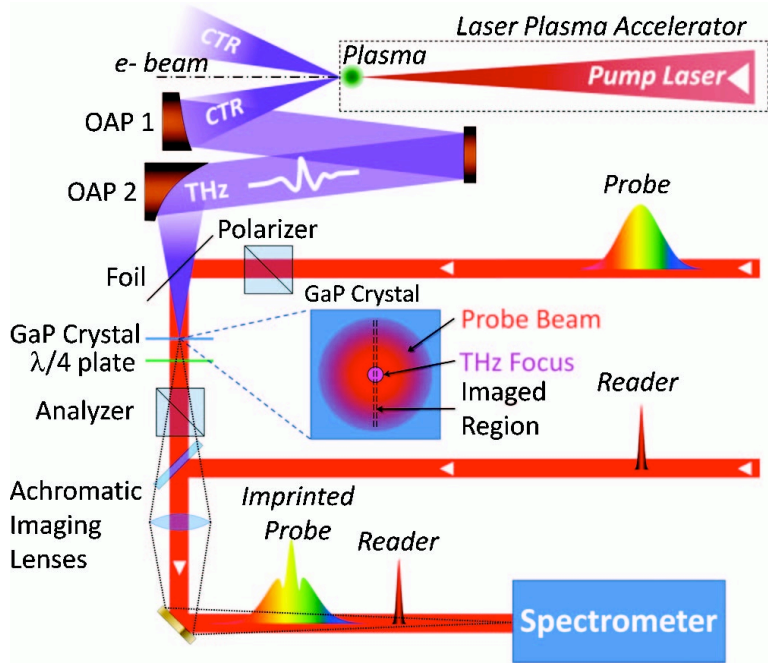

Fig. 1. Schematic of the TEX detection scheme. beam of $\mathrm{THz}$ was primarily linearly polarized, with a residual transversely polarized component analytically estimated to be at the $10 \%$ level. The $\mathrm{THz}$ beam was then refocused with a second OAP onto a $200 \mu \mathrm{m}$ thick gallium phosphide (GaP) crystal cut along $\langle 1,1,0\rangle$. An optical pulse split from the pump beam that generated the electrons was overcompressed, resulting in a temporally chirped pulse ("probe") with a FWHM duration of 2 ps. A second fully compressed optical pulse ("reader"), measured to have a FWHM duration of $45 \mathrm{fs}$, was also split from the pump beam. The probe and the THz pulses were overlapped colinearly in the GaP crystal, resulting in the EO interaction. A polarizer was used to purify the polarization state of the incident probe, and a second polarizer (referred to as an "analyzer") was used to convert polarization rotations into amplitude modulations. A quarter wave plate was used to offset the transmission to the $50 \%$ level, allowing both positive and negative $\mathrm{THz}$ fields to be resolved. The transmitted probe pulse was then combined colinearly with, but temporally offset from, the reader and sent into a $0.27 \mathrm{~m}$ imaging spectrometer with a 14 bit, 1 MP cooled CCD detector, producing a spectral interferogram image ("TEXogram"). A pair of achromatic lenses was used to image the interaction plane to the input plane of the spectrometer, where the spectometer slit is used to select a spatiotemporal slice of the probe.

The choice to encode the THz signal onto either the amplitude or the phase of the probe field is made by appropriately choosing the polarization state of the incident probe (Fig. 2). If the polarization is aligned at $45^{\circ}$ to the principal axes of the THz-induced index ellipsoid, the probe polarization will be rotated in proportion to the $\mathrm{THz}$ field strength [27,28], but the phase-shift contribution from each of the axes will cancel, resulting in pure amplitude modulation [Fig. 2(a)]. However, if the probe polarization is aligned along one of the principal axes, the probe will experience a temporally varying phase shift, but no polarization rotation, resulting in pure phase modulation [Fig. 2(b)]. For other polarization states, the $\mathrm{THz}$ imprint is mixed between phase and amplitude with a strongly nonlinear dependence on the field strength, making waveform retrieval unreliable.
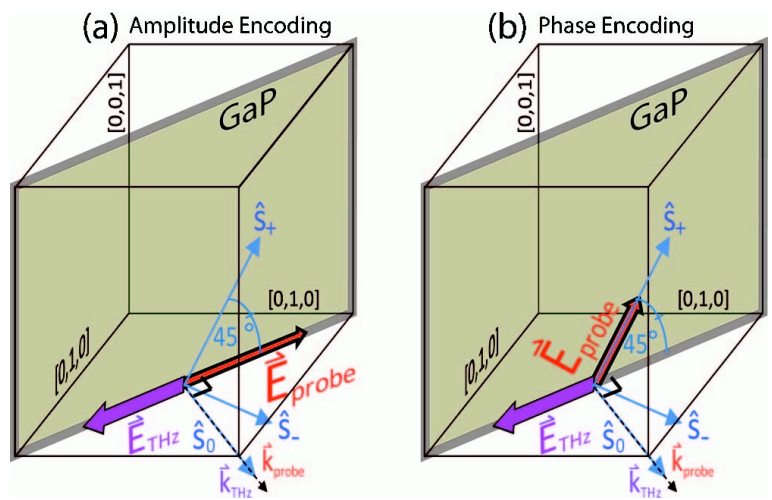

Fig. 2. (a) Amplitude-encoding geometry. The probe polarization is aligned between the principal axes $\left(\hat{S}_{+}, \hat{S}_{-}\right)$of the THz-induced birefringence, resulting in polarization rotation only. (b) Phase-encoding geometry. The probe polarization is parallel with one of the principal axes, resulting in phase shifting only. 


\section{ANALYSIS}

The recovery of THz waveform occurs in two distinct steps. The first step is the recovery, postinteraction, of the spatiotemporal electric field of the probe, which can be written $E_{p}(y, t) \equiv \mathcal{E}(y, t) \exp \{i \Phi(y, t)\}$, where $t$ is time, $y$ is the spatial coordinate, and $\mathcal{E}(y, t)$ and $\Phi(y, t)$ are the probe's temporal amplitude and phase, respectively. The second step is the determination of the $\mathrm{THz}$ waveform spatiotemporal profile, $E_{\mathrm{THz}}(y, t)$, from the THz-induced modulations of either the probe amplitude or the probe phase, depending on the encoding method.

In the first step, $E_{p}(y, t)$ is obtained from the TEXogram by applying a single one-dimensional FT operation independently to each row of the spectral image. The TEXogram is described by $T_{\mathrm{EX}}(y, \omega)=\left|\tilde{E}_{p}(y, \omega)\right|^{2}+\left|\tilde{E}_{r}(y, \omega)\right|^{2}+\tilde{E}_{p}(y, \omega) \tilde{E}_{r}^{*}(y, \omega)+$ c.c., where $\omega$ is the optical angular frequency and $\tilde{E}_{p}(y, \omega)$ and $\tilde{E}_{r}(y, \omega)$ are the electric fields of the probe and reader pulses, respectively, in the spectral domain. The FT results in a time-domain signal containing four components corresponding to the four terms of the TEXogram. The first two terms of $T_{\mathrm{EX}}(y, \omega)$ produce overlapping peaks known as "coherence spikes" at $t=0$, while the third and fourth terms (i.e., "cross terms"), which are responsible for the fringes in the TEXogram, produce side peaks at $t= \pm \Delta t$, where $\Delta t$ is the delay of the probe behind the reader (Fig. 3). The "convolution theorem" of FTs identifies the FT of the cross terms with the complex cross correlation of the electric fields of the probe and the reader in the time domain; i.e., if the FT is expressed as $\operatorname{FT}\left[T_{\mathrm{EX}}(y, \omega)\right](t)=A(y, t)+C(y, t)+C^{*}(y,-t)$, where $A(y, t)$ is the FT of the first two terms of $T_{\mathrm{EX}}(y, \omega)$ and $C(y, t) \equiv \mathrm{FT}\left[\tilde{E}_{p}(y, \omega) \tilde{E}_{r}^{*}(y, \omega)\right](t)$ is the FT of the first cross term, then $C(y, t)=\int_{-\infty}^{\infty} E_{p}(y, \tau) E_{r}^{*}(y, \tau-t) \mathrm{d} \tau$ is the cross correlation of the probe temporal field given by $E_{p}(y, t)=$ $\operatorname{FT}\left[\tilde{E}_{p}(y, \omega)\right](t)$ with the reader temporal field given by $E_{r}(y, t)=\mathrm{FT}\left[\tilde{E}_{r}(y, \omega)\right](t)$. For a reader with a suitably short duration and negligible spectral phase, the side peak in the FT of $T_{\mathrm{EX}}(\omega, y)$ approximates the chirped probe pulse in both amplitude and phase: $C(y, t) \approx E_{p}(y, t)$.

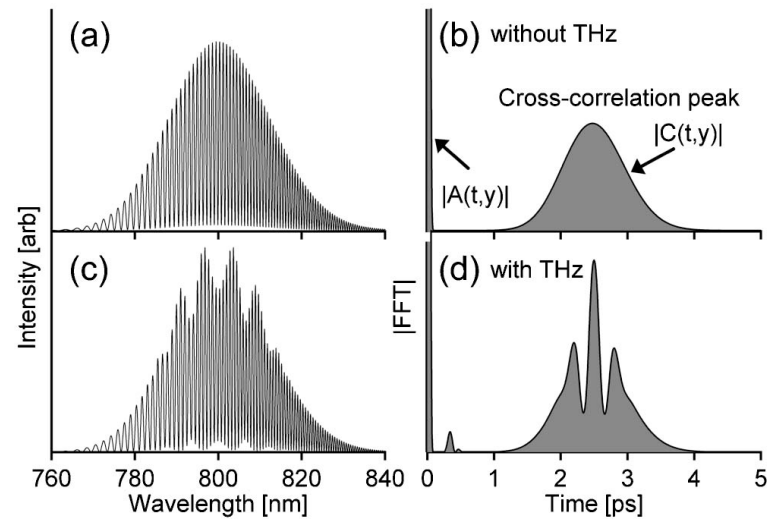

Fig. 3. (a) Simulated TEX interferogram in the absence of $\mathrm{THz}$ showing interference in the spectrometer between copropagating probe and reader pulses separated in time by $2.5 \mathrm{ps}$. (b) Modulus of the FT of the interferogram in (a) showing a broad side peak at $2.5 \mathrm{ps,}$ which represents the amplitude of the cross correlation between the probe and reader electric fields. (c) Simulated TEX interferogram with $\mathrm{THz}$ present. (d) Modulus of the complex FT of the interferogram in (c), showing a THz-induced modulation in the side peak amplitude.
In the second step, the $\mathrm{THz}$ waveform is recovered by determining the modulation to either $\mathcal{E}(y, t)$ or $\Phi(y, t)$. In the amplitude-encoding method, the transmitted probe amplitude is given by $\mathcal{E}_{T}^{2}(y, t)=\frac{1}{2} \mathcal{E}_{0}^{2}(y, t)\left[1+\sin \left(\alpha E_{\mathrm{THz}}(y, t)\right)\right]$, where $\alpha \equiv 2 \pi \frac{L}{\lambda} n_{0}^{3} r_{41}, L$ is the crystal thickness, $\lambda$ is the optical wavelength, $n_{0}$ is the linear index in the absence of THz, $r_{41}$ is the linear EO coefficient, and the 0 and $T$ subscripts denote field quantities before and after the interaction, respectively. In the phase-encoding method, the transmitted phase is given by $\Phi_{T}(y, t)=\Phi_{0}(y, t) \pm \alpha E_{\mathrm{THz}}(y, t) / 2$, where the sign depends on the choice of principal axis. As $\mathcal{E}_{0}(y, t)$ and $\Phi_{0}(y, t)$ are not simultaneously acquired in TEX, a separate ("null") shot in the absence of $\mathrm{THz}$ is required. The reconstructed waveform is thus given by $E_{\mathrm{THz}}(y, t)=$ $\alpha^{-1} \arcsin \left[\mathcal{E}_{T}^{2}(y, t) / \mathcal{E}_{\text {null }}^{2}(y, t)-1\right]$ for amplitude encoding and $E_{\mathrm{THz}}(y, t)=2 \alpha^{-1}\left[\Phi_{T}(y, t)-\Phi_{\text {null }}(y, t)\right]$ for phase encoding.

The temporal resolution of the $\mathrm{THz}$ waveform retrieval is determined by two independent factors: the duration of the reader, which determines the temporal resolution of the probe electric-field recovery, and the thickness of the EO crystal, which determines the EOS phase-matching bandwidth. Calculations simulating the probe field retrieval process in both phase- and amplitude-encoding configurations for a $45 \mathrm{fs}$ reader confirm accurate reconstruction of the encoded signal for frequencies beyond $\sim 8 \mathrm{THz}$, which is the resolution limit set by the $200 \mu \mathrm{m} \mathrm{GaP}$ crystal. The limitation to the temporal range of the detection is set by the bandwidth and spectral resolution of the imaging spectrometer, which must be capable of resolving the spectral fringes over the full bandwidth of the probe.

\section{RESULTS}

Figure 4 shows a sample THz spatiotemporal waveform image acquired using TEX in the amplitude-encoding configuration.
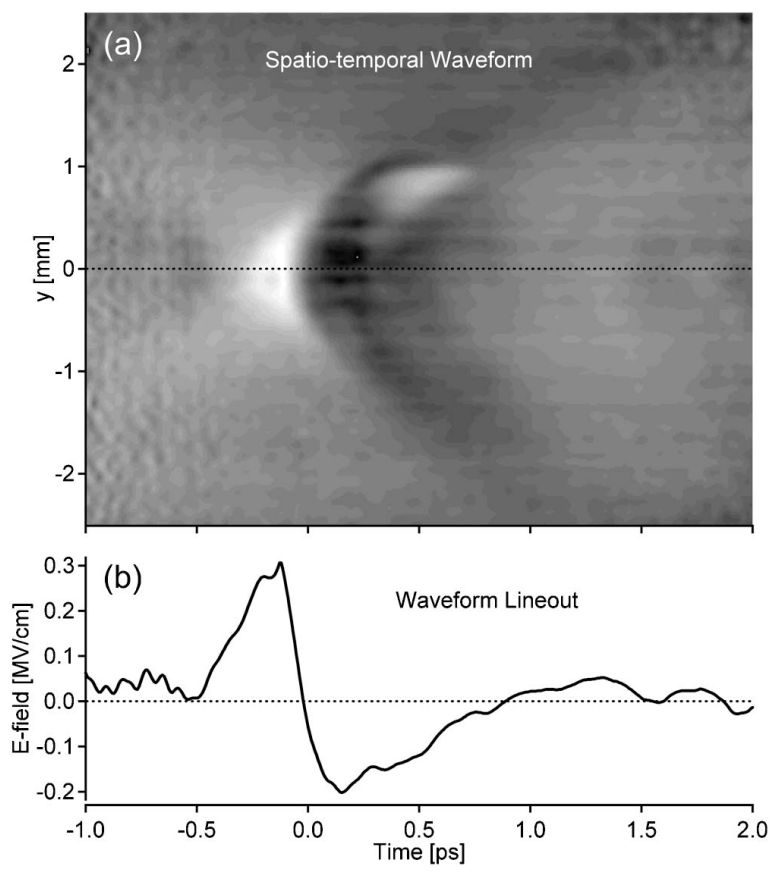

Fig. 4. Data showing (a) $\mathrm{THz}$ spatiotemporal waveform extracted from the raw interferogram and (b) lineout of waveform at $y=0 \mathrm{~mm}$. 

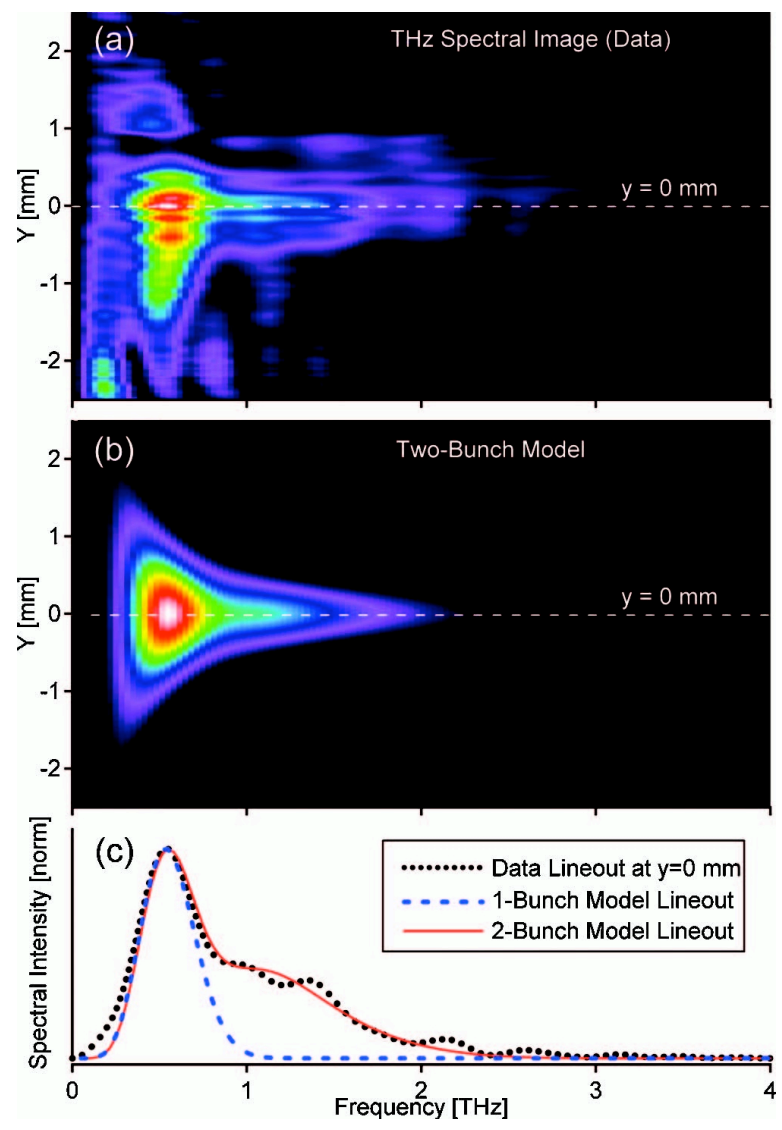

Fig. 5. (a) Power spectrum of THz waveform in Fig. 4. (b) Spectral image calculated using a two-bunch model. (c) Lineouts of spectral images for data (black dotted line), a one-bunch model (blue dashed line), and a two-bunch model (red solid line). Comparison shows that a two-bunch model yields a significantly better fit than a one-bunch model.

The measured waveform is nearly single cycle and displays sharp temporal features of order $100 \mathrm{fs}$, illustrating the need for high temporal resolution. The waveform also exhibits strong spatiotemporal coupling, in the shape of an " $\mathrm{X}$," as was described by Jiang and Zhang [20], which can be understood in terms of a variation of the Gouy phase shift and focused-waist size with wavelength.

To diagnose the structure of the electron bunch, the spectrum of the THz waveform shown in Fig. 4 was calculated and compared with theory. The spatial and temporal features of the spectral image were modeled (Fig. 5) by using CTR emission theory [26] with the inclusion of collection and propagation effects. To accurately model both the low- and highfrequency parts of the spectral image, two bunches of different duration and charge ( $90 \%$ of the charge in a $140 \mu \mathrm{m}-\mathrm{rms}$ bunch and $10 \%$ in a $50 \mu \mathrm{m}$ bunch) were required. Comparison of a one-bunch model (containing only the longer bunch) with the two-bunch model shows that the contribution to $\mathrm{THz}$ emission above $\sim 0.75 \mathrm{THz}$ comes entirely from the shorter bunch, despite its small relative charge [Fig. 5(c)]. This high sensitivity to the presence of the shorter electron bunch is an important confirmation of the practicality of the THz-based diagnostic for characterizing LPAs, since the high-energy, low-charge electron-bunch component of interest is often accompanied by a lower-energy component containing the bulk of the charge. The electron energy spectrum, measured simultaneously, does, in fact, show a two-component distribution with a large thermal component and a smaller "quasimonoenergetic" component. The importance of recovering the spatial variations in the $\mathrm{THz}$ waveform is illustrated by the strong spatial dependence of the spectrum of the focused $\mathrm{THz}$ pulse: the higher-frequency component from the short bunch is more localized to the axis. A spatially integrated technique would under-represent this component, thus diminishing sensitivity to the presence of the short bunch. In addition, because the transverse focal size of a given spectral component is strongly dependent on not only the wavelength but also the spectrally varying far-field intensity distribution, the nice correspondence between the data and model of the shape of the spectral image provides a confirmation of the $\mathrm{THz}$ emission patterns predicted by CTR theory.

\section{CONCLUSION}

A new single-shot technique (TEX) is demonstrated for measurement of ultrafast phenomena resulting in temporal phase or amplitude modulations. TEX provides high temporal resolution and one-dimensional imaging simultaneously for the first time in EOS, enabling analysis of spatiotemporal and spatiospectral coupling in $\mathrm{THz}$ waveforms. TEX is significantly easier to set up than previous high-resolution, single-shot EO techniques because it does not require the use of nonlinear processes other than the linear Pockels effect used for EOS. Because TEX is linear in the probe field strength, low-power, low-cost, unamplified laser systems may be used, making it highly accessible. In addition, its dual phase- and amplitude-encoding capability makes it applicable to a wide range of phenomena. The temporal resolution is set by the duration of the reader pulse, and can be made smaller than the intrinsic limitation set by phase matching in the EOS process. The single-shot temporal detection window is tunable in the range of several to tens of picoseconds and limited by the bandwidth and spectral resolution of the spectrometer. Numerical analysis confirms the capability of TEX to reproduce THz waveforms in both temporal and spectral domains without significant distortion in both phase- and amplitude-encoding configurations. Waveforms of $\mathrm{THz}$ pulses generated as CTR from an LPA were measured using the amplitude-encoding configuration and analyzed. The resultant $\mathrm{THz}$ spectral images were used to demonstrate the presence of heterogeneous electron-bunch structure from the LPA. The dependence of the THz spectrum on the electron-bunch duration makes it possible to detect the presence of short electron-bunch substructure with high sensitivity.

\section{ACKNOWLEDGMENTS}

The authors acknowledge Carl B. Schroeder, Kei Nakamura, Cameron G. R. Geddes, Anthony J. Gonsalves, Csaba Tóth, and Eric H. Esarey for their valuable contributions. This work was supported by Defense Advanced Research Projects Agency (DARPA) and by the Director, Office of Science, Office of High Energy Physics, of the United States Department of Energy (DOE) under contract DE-AC02-05CH11231. 


\section{REFERENCES}

1. J. van Tilborg, "Coherent terahertz radiation from laserwakefield-accelerated electron beams," Ph.D. dissertation (Technische Universiteit Eindhoven, 2006).

2. Q. Wu and X.-C. Zhang, "Free-space electro-optic sampling of terahertz beams," Appl. Phys. Lett. 67, 3523-3525 (1995).

3. Q. Wu, T. Hewitt, and X.-C. Zhang, "Two-dimensional electrooptic imaging of THz beams," Appl. Phys. Lett. 69, 1026-1028 (1996).

4. A. Nahata, D. H. Auston, and T. F. Heinz, "Coherent detection of freely propagating terahertz radiation by electro-optic sampling," Appl. Phys. Lett. 68, 150-152 (1996)

5. R. A. Kaindl, R. Huber, B. A. Schmid, M. A. Carnahan, D. Hägele, and D. S. Chemla, "Ultrafast thz spectroscopy of correlated electron: from excitons to cooper pairs," Phys. Stat. Sol. (b) 243, 2414-2422 (2006).

6. M. C. Beard, G. M. Turner, and C. A. Schmuttenmaer, "Transient photconductivity in gaAs as measured by time-resolved terahertz spectroscopy,” Phys. Rev. B 62, 15764-15777 (2000).

7. M. C. Hoffmann, J. Hebling, H. Y. Hwang, K.-L. Yeh, and K. A Nelson, "THz-pump/THz-probe spectroscopy of semiconductors at high field strengths," J. Opt. Soc. Am. B 26, A29-A34 (2009).

8. J. van Tilborg, C. B. Schroeder, C. V. Filip, C. Tóth, C. G. R Geddes, G. Fubiani, R. Huber, R. A. Kaindl, E. Esarey, and W. P. Leemans, "Temporal characterization of femtosecond laser-plasma-accelerated electron bunches using terahertz radiation," Phys. Rev. Lett. 96, 014801 (2006)

9. Q. Wu and X.-C. Zhang, "Ultrafast electro-optic field sensors," Appl. Phys. Lett. 68, 1604-1606 (1996).

10. Z. Jiang and X.-C. Zhang, "Electro-optic measurement of THz field pulses with a chirped optical beam," Appl. Phys. Lett. 72, 1945-1947 (1998).

11. Z. Jiang and X.-C. Zhang, "Single-shot spatiotemporal terahertz field imaging," Opt. Lett. 23, 1114-1116 (1998).

12. F. G. Sun, Z. Jiang, and X.-C. Zhang, "Analysis of terahertz pulse measurement with a chirped probe beam,” Appl. Phys. Lett. 73, 2233-2235 (1998).

13. J. Fletcher, "Distortion and uncertainty in chirped pulse $\mathrm{THz}$ spectrometers," Opt. Express 10, 1425-1430 (2002).

14. S. Jamison, J. Shen, A. M. MacLeod, W. A. Gillespie, and D. A. Jaroszynski, "High-temporal-resolution, single-shot characterization of terahertz pulses," Opt. Lett. 28, 1710-1712 (2003).

15. J. van Tilborg, C. B. Schroeder, C. Tóth, C. G. R. Geddes, E. Esarey, and W. P. Leemans, "Single-shot spatiotemporal mea- surements of high-field terahertz pulses," Opt. Lett. 32, 313-315 (2007).

16. U. Schmidhammer, V. De Waele, J.-R. Marqus, N. Bourgeois, and M. Mostafavi, "Single shot linear detection of $0.0110 \mathrm{THz}$ electromagnetic fields: electro-optic sampling with a supercontinuum in balanced detection,” Appl. Phys. B 94, 95-101 (2009).

17. I. Wilke, A. MacLeod, W. Gillespie, G. Berden, G. Knipples, and A. van der Meer, "Single-shot electron-beam bunch length measurements," Phys. Rev. Lett. 88, 124801 (2002).

18. X. Yan, A. MacLeod, W. Gillespie, G. Knippels, D. Oepts, A. van der Meer, and W. Seidel, "Subpicosecond electro-optic measurement of relativistic electron pulses," Phys. Rev. Lett. 85, 3404-3407 (2000)

19. X.-Y. Peng, O. Wiili, M. Chen, and A. Pukhov, "Optimal chirped probe pulse length for terahertz pulse measurement," Opt Express 16, 12342-12349 (2008).

20. Z. Jiang and X.-C. Zhang, "2D measurement and spatio-temporal coupling of few-cycle THz pulses," Opt. Express 5, 243-248 (1999).

21. W. P. Leemans, C. G. R. Geddes, J. Faure, C. Tóth, J. van Tilborg, C. B. Schroeder, E. Esarey, G. Fubiani, D. Auerbrach, B. Marcelis, M. A. Carnahan, R. A. Kaindl, J. Byrd, and M. C. Martin, "Observation of terahertz emission from a laserplasma-accelerated electron bunch crossing a plasma-vacuum boundary," Phys. Rev. Lett. 91, 074802 (2003).

22. W. P. Leemans, J. van Tilborg, J. Faure, C. G. R. Geddes, C. Tóth, C. B. Schroeder, E. Esarey, G. Fubiani, and G. Dugan, "Terahertz radiation from laser accelerated electron bunches," Phys. Plasmas 11, 2899-2906 (2004).

23. S. P. LeBlanc, E. Gaul, N. H. Matlis, A. Rundquist, and M. C. Downer, "Single-shot ultrafast phase measurement by frequency domain holography,” Opt. Lett. 25, 764-766 (2000).

24. N. H. Matlis, S. Reed, S. S. Bulanov, V. Chvykov, G. Kalintchenko, T. Matsuoka, P. Rousseau, V. Yanovsky, A. Maksimchuk, S. Kalmykov, G. Shvets, and M. C. Downer, "Snapshots of laser wakefields," Nature Phys. 2, 749-753 (2006).

25. K. Kim, I. Alexeev, and H. Milchberg, "Single-shot supercontinuum spectral interferometry," Appl. Phys. Lett. 81, 4124-4126 (2002).

26. C. B. Schroeder, E. Esarey, J. van Tilborg, and W. P. Leemans, "Theory of coherent transition radiation generated at a plasmavacuum interface,” Phys. Rev. E 69, 016501 (2004).

27. R. Boyd, Nonlinear Optics (Academic, 1992).

28. G. Gallot and D. Grischkowsky, "Electro-optic detection of terahertz radiation,” J. Opt. Soc. Am. B 16, 1204-1212 (1999). 